\title{
Laboratory perspectives: Population biology and the 'new' enterococcus
}

\author{
WENDY M JOHNSON PhD, MICHAEL B COULTHART PhD
}

M ost major hospitals in Canada have well established and effective infection control programs to identify and respond quickly to alert organisms, which are most likely to give rise to outbreaks. Some of the organisms most worrisome to clinicians are those that are resistant to one or more antimicrobials (1). An example of alert condition surveillance for antibiotic resistant bacteria is the systematic laboratory-based ward screening for methicillin-resistant Staphylococcus aureus (MRSA). Unfortunately, only a minority of nosocomial infections can be averted by this type of surveillance and, in order to improve overall surveillance and to enhance the potential for better prevention of infection, additional strategies are required as an adjunct to alert organism survey. Such targeted surveillance in defined subgroups of patients is conducted frequently in high priority/high risk areas such as intensive care, transplantation, renal dialysis, burn and oncology units.

In late 1995 , the medical community in Canada witnessed an outbreak of patient colonization with vancomycin resistant Enterococcus faecium expressing the VanB phenotype (see reference 2 for a recent review of glycopeptide resistance in enterococci) at the Toronto Hospital. A team led by Dr John Conly identified the resistant organism in 39 patients during targeted surveillance in a renal dialysis unit (3). Fortunately, in this incident only a single patient was actually infected and required treatment with teicoplanin. The outbreak has since been brought under control. Nevertheless, vancomycin resistant enterococci (VRE), chiefly represented by E faecium and Enterococcus faecalis, are becoming recognized as pre-eminent nosocomial pathogens of this decade in tertiary care hospitals, in both the United States and the United Kingdom

Bureau of Microbiology, Laboratory Centre for Disease Control, Ottawa, Ontario

Correspondence and reprints: Dr WM Johnson, Room 240, HPB Building, Tunney's Pasture, Ottawa, Ontario K1A OL2. Telephone 613-957-1356, fax 613-941-2408, e-mailwjohnson@hpb.hwc.ca
(4-6). This recent occurrence in Canada emphasizes that a constant state of readiness is essential in order to quickly identify these organisms and to implement appropriate infection control precautions.

Little is known about the origin and spread of VRE in Canadian hospitals that could be usefully applied to its prevention and control. At the same time, these organisms may cause life-threatening infections in some cases, especially those associated with expression of the VanA phenotype (2), for which few effective therapies are available. For these reasons, hospital infection control committees are being encouraged to discuss this problem and to review the recommendations of the Hospital Infection Control Practices Advisory Committee for preventing the spread of vancomycin resistance (7). Restricted use of vancomycin, rigorous infection control and continued vigilance appear to be the best options until we have a better understanding of the origin and spread of VRE. A recent editorial (8) summarized the initiatives in place to prevent the spread of VRE and expressed concern about the possible spread of resistance factors to other mulitresistant nosocomial pathogens such as MRSA. In the same issue of Clinical Infectious Diseases, Sydney Finegold (9) included a special editorial message, pointing out that enterococci are now the second most common cause of nosocomial infection next to Escherichia coli.

In order to collect point-prevalence data on VRE in major Canadian hospitals to promote a national perspective, the Laboratory Centre for Disease Control (LCDC) is supporting, in collaboration with the Canadian Hospital Epidemiology Committee (CHEC), a survey as part of its Canadian Nosocomial Infection Surveillance Program (CNISP). The history and scope of this program, in addition to others, were reviewed in a recent LCDC Report (10). The one-month survey was completed in early February 1996 and included six categories of high risk bed units. A total of 27 hospitals participated in the study and all VRE isolates collected will be fully characterized using a variety of phenotypic and genotypic methods in several participating laboratories. 


\section{STUDYING THE ORIGIN AND SPREAD OF NOSOCOMIAL VRE}

It is becoming more important to understand, in biochemical, genetic, ecological and evolutionary terms, the biological processes leading to emergence and spread of antimicrobial resistance phenotypes in bacterial populations. This knowledge is likely to play a key role in the development of practical solutions to problems of prevention and control of resistance, both by serving as a consensus scientific framework for discussion and, eventually, by revealing the most effective points for intervention in the cycles of microbial colonization, infection, reproduction and transmission that underlie the population dynamics of resistance. Such an approach will ultimately define and serve the needs of infectious disease 'surveillance' in the largest sense, where data describing spatial and temporal patterns of incidence and prevalence of specific kinds of infections (eg, those caused by VRE) are made more deeply intelligible by drawing on a detailed understanding of the population biology of the pathogen in specific settings.

An application of this approach comes from the 1996 CNISP VRE Point-Prevalence Survey mentioned above. In the CNISP survey, in addition to submitting any resistant isolates discovered during the survey period, the participating hospitals agreed to collect a broader sample of vancomycin susceptible enterococcal (VSE) isolates from up to six high risk bed units per hospital. These 'background' strain collections can be used (pending approval of and consensus among CHEC members) to address certain biological questions, for which a sample consisting only of resistant strains would be inadequate. Such questions fall into two basic categories, as explained below.

First, very few details are known about the ecology of transmission of enterococci in hospital environments. It is known that enterococci (especially $E$ faecium and $E$ faecalis) are essentially ubiquitous human commensals, and that they tend to be environmentally hardy and thus highly likely to be capable of direct or indirect transmission through casual contact or by fomites. Beyond this, however, there are very few data on, for instance, how commonly new enterococcal strains are acquired by patients in various hospital settings (eg, intensive care units, oncology wards, transplant units) during their stay in hospital, or the patterns of strain sharing within and between bed units, hospitals, communities and geographic regions. Such information is not easily acquired, since it requires not only systematic sampling of patients' enterococcal flora in a spatially and temporally defined manner with standardized methods, but also sophisticated methods for measurement and statistical analysis of genetic relationships between sampled strains. The first of these requirements was fulfilled with the cooperation of participants in the CNISP VRE Point-Prevalence Survey, who were willing to collect VSE strains recovered from their hospitals during the survey period. (It is incidental, from the standpoint of the more general biological question asked above, that the sampling locations and time period are coincident with the four-week VRE PointPrevalence Survey.)

The second set of requirements can ultimately be fulfilled only by DNA sequence-based phylogenetic analysis of entero- coccal strain lineage relationships. In this connection, recently it has become clear (11-14) that bacterial populations, to varying and unpredictable degrees, are highly prone to parasexual 'horizontal transfer' of genetic material, causing their chromosomal DNA to comprise a mosaic of such horizontally transferred segments and other segments inherited 'vertically' from immediate ancestors in the usual manner. This mosaicism creates many theoretical and practical complications for interpretation of genetic relationships between bacterial strains belonging to moderately distant reproductive lineages. Specifically, when two strains are not genetically identical, the vertically inherited segments shared by the strains are the only ones that can yield a consistent and accurate picture of lineage relationships, and therefore help in understanding transmission patterns, but such vertically inherited segments are by no means easy to identify a priori. With commonly used 'strain-typing' strategies, such as pulsed-field gel electrophoresis or multilocus enzyme electrophoresis, which afford only a relatively sparse sampling of the bacterial chromosome and produce only a single binary or at best multistate data set for each sampled locus, it is impossible to know which subset of markers at polymorphic loci has descended vertically and which has been transferred horizontally. The only kind of genetic data with the power to overcome such difficulties is DNA sequence or high density restriction maps, recovered from a sample of dispersed chromosomal loci that is large enough to establish a consensus phylogenetic tree and therefore to estimate more definitely strain lineage relationships. Such a study, although by now carried out repeatedly for well studied organisms such as $E$ coli $(12,13)$, has never been published for any Enterococcus species. Exciting opportunities exist to carry out such studies on the VRE-VSE collection gathered from the CHEC-CNISP collaboration.

The second kind of biological question that may be addressed with DNA sequence data from a general sample of enterococcal strains concerns the origins of VRE in relation to the VSE background. It is widely thought that the most commonly occurring VanA and VanB phenotypes of VRE depend on the presence of clusters of genes found on transposons; these transposons are capable of integrating into conjugative plasmids and therefore, in turn, of being transferred between enterococcal lineages (15). Thus, understanding the population dynamics of VRE will require us to understand the (largely independent) population dynamics of, first, these two types of mobile DNA elements; second, the enterococcal chromosome; and third, the cell lineages that carry them, as well as interactions among these three phenomena.

Genetic analysis of lineage relationships between VRE and VSE strains recovered during the CNISP survey at a similar time from the same patient, the same bed unit or the same hospital may afford a unique opportunity to gain insight into the specific circumstances surrounding the origin and spread of VRE. For instance, it may be the case that most VRE found in a given hospital at a given time, including those that may be recovered from 'healthy carriers', are more closely related to each other than they are to strains belonging to the VSE background in the same hospital, suggesting 'epidemic 
spread' of strains. Alternatively, a vancomycin resistance phenotype may be associated with a diversity of chromosomal backgrounds representing many phylogenetic lineages, suggesting perhaps extensive lateral transfer of mobile elements carrying genetic determinants of vancomycin resistance. These are just two simple scenarios among many possible patterns of relationship between VRE and VSE; of the two types of biological questions outlined here, this is definitely the more complex.

The serious challenge presented to Canadian health care providers by the recent emergence and potential spread of VRE justifies a coordinated, prospective, multifaceted approach, enlisting the efforts of members of hospital and government diagnostic laboratories, infection control specialists, epidemiologists and research scientists. Although not an integral part of a point-prevalence survey for resistant strains, contemporaneous collection of a general sample of enterococci from hospitalized patients has a strong potential to yield important benefits through a more detailed scientific understanding of the dynamics of strain transmission and resistance in enterococcal populations. Finally, the results of these studies on enterococci may help to formulate a more widely applicable methodological model for the study of antimicrobial resistance in other nosocomial pathogens.

ACKNOWLEDGEMENTS: The authors acknowledge close collaborations with Shirley Paton, Marianna Ofner and Irene Martin (LCDC), and the continuing support and cooperation of the CHEC VRE Working Group.

\section{REFERENCES}

1. Armstrong D, Neu H, Peterson LR, Tomasz A. The prospects of treatment failure in the chemotherapy of infectious diseases in the 1990s. Microb Drug Resist 1995;1:1-4.

2. Woodford N, Johnson AP, Morrison D, Speller DCE. Current perspectives on glycopeptide resistance. Clin Microbiol Rev 1995;8:585-615.

3. Lior L, Litt M, Conly J, Hockin J. Vancomycin-resistant enterococci arrives in Canada. Epidemiology Intelligence Service Conference. Atlanta, April 1996. (Abst)

4. Spera RV Jr, Farber BF. Multiply-resistant Enterococcus faecium: the nosocomial pathogen of the 1990s. JAMA 1992;268:2563-4.

5. Centers for Disease Control. Nosocomial enterococci resistant to vancomycin - United States, 1989-1993. MMWR 1993;42:597-9.

6. PHLS. Vancomycin resistant enterococci in hospitals in the United Kingdom. Wkly Commun Dis Rep 1995;50:281-4.

7. Hospital Infection Control Practices Advisory Committee (HICPAC). Recommendations for preventing the spread of vancomycin resistance. MMWR 1995;44:1-12.

8. Murray BE. Editorial response: What can we do about vancomycin-resistant enterococci? Clin Infect Dis 1995;20:1134-6. (Edit)

9. Finegold SM. A special message from the editor. Clin Infect Dis 1995;20:1091. (Edit)

10. Paton S. Nosocomial infection program. Can J Infect Dis 1995;6:73-5.

11. Maynard Smith J, Smith NH, O'Rourke MO, Spratt BG. How clonal are bacteria? Proc Natl Acad Sci USA 1993;90:4384-8.

12. Milkman R, Bridges M. Molecular evolution of the Escherichia coli chromosome. IV. Sequence comparisons. Genetics 1993; 133:455-68.

13. Guttman DS, Dykhuizen DE. Clonal divergence in Escherichia coli as a result of recombination, not mutation. Science 1994;266:1380-3.

14. Lan R, Reeves PR. Gene transfer is a major factor in bacterial evolution. Mol Biol Evol 1996;13:47-55.

15. Arthur M, Courvalin P. Genetics and mechanisms of glycopeptide resistance in enterococci. Antimicrob Agents Chemother 1993;37:1563-71. 


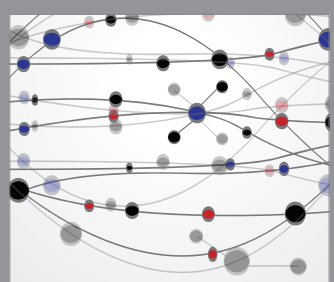

The Scientific World Journal
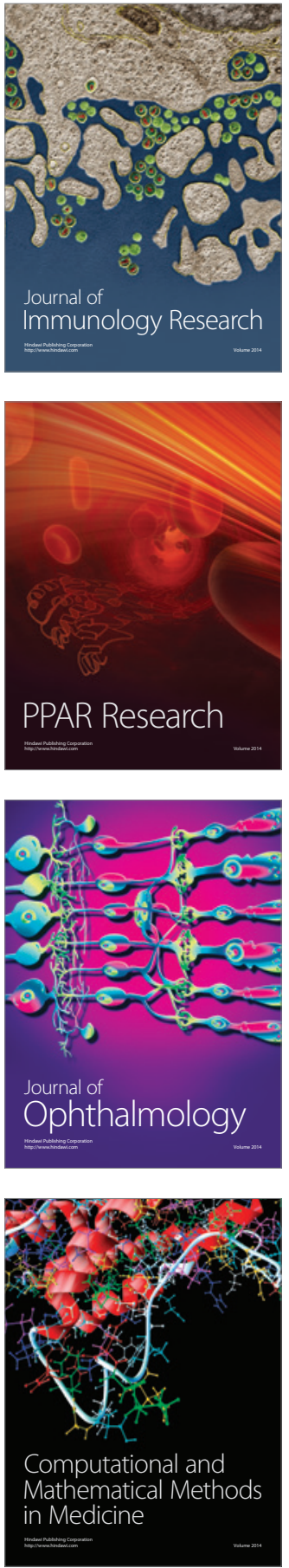

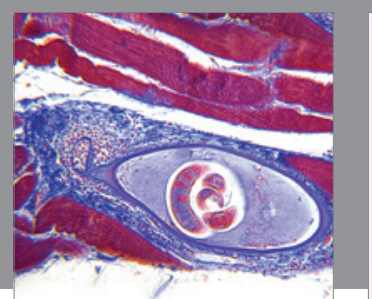

Gastroenterology Research and Practice

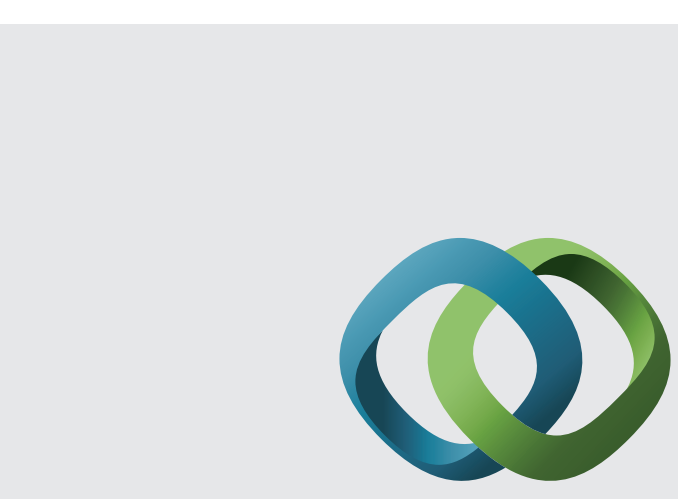

\section{Hindawi}

Submit your manuscripts at

http://www.hindawi.com
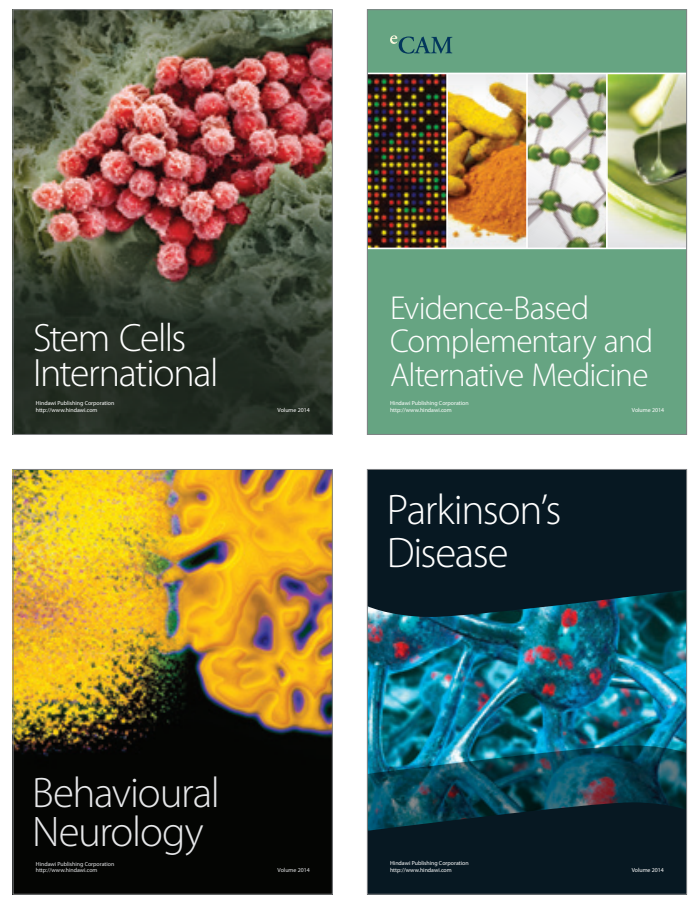
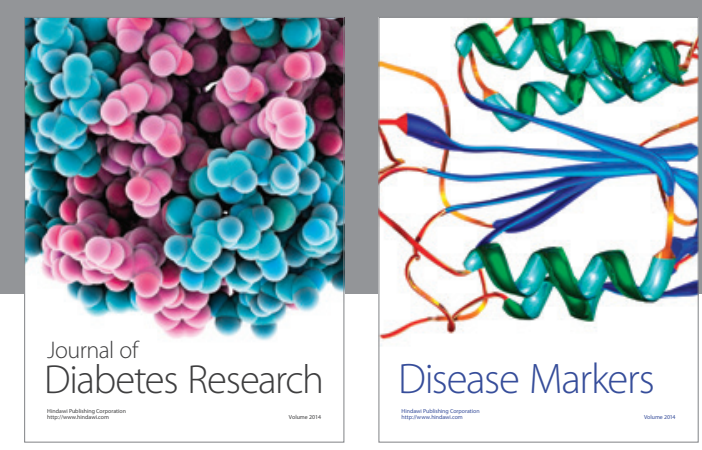

Disease Markers
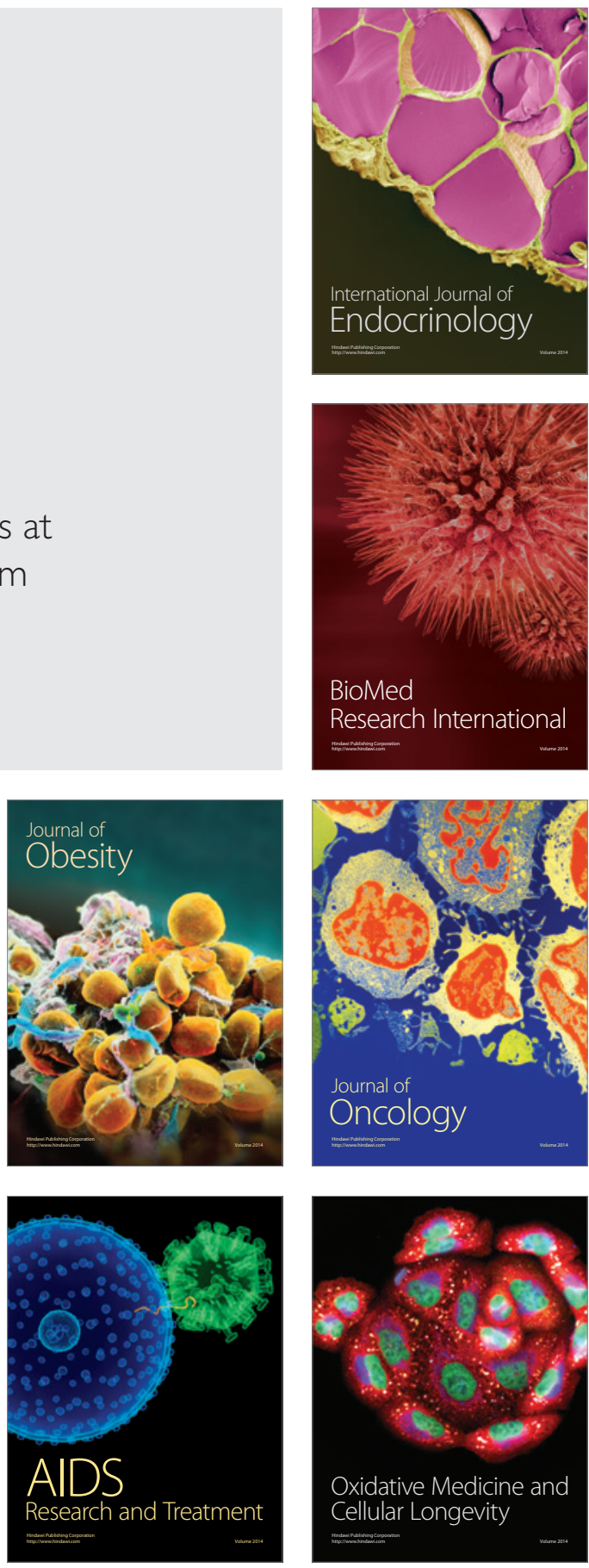